L'HOMME L'Homme

Revue française d'anthropologie

169 | 2004

Varia

\title{
Identité et métier des inaden
}

La valeur sociale des techniques chez les Touaregs de l'Ouest

\section{Catherine Hincker}

\section{(2) OpenEdition \\ Journals}

Édition électronique

URL : http://journals.openedition.org/lhomme/21580

DOI : 10.4000//homme.21580

ISSN : 1953-8103

Éditeur

Éditions de l'EHESS

Édition imprimée

Date de publication : 1 février 2004

Pagination : 127-151

ISSN : 0439-4216

Référence électronique

Catherine Hincker, «Identité et métier des inaden », L'Homme [En ligne], 169 | 2004, mis en ligne le 01 janvier 2006, consulté le 21 avril 2019. URL : http://journals.openedition.org//homme/21580 ; DOI : 10.4000//homme.21580 


\title{
Identité et métier des inaḍen \\ La valeur sociale des techniques chez les Touaregs de l'Ouest
}

\author{
Catherine Hincker
}

L A DIFFICULTÉ à traduire le terme inaden en français est à l'origine de cet article. Nombre d'auteurs, spécialistes du monde touareg, retiennent, pour désigner ce groupe, la traduction française "artisans" ou "forgerons $»^{1}$. Tous reconnaissent que cette traduction est retenue faute de mieux car elle ne recouvre que partiellement la réalité. En effet, ces deux termes mettent essentiellement l'accent sur la pratique d'une activité technique, or les inaden n'exercent pas tous un métier. S'ils ne sont pas tous "artisans ", ils jouent en revanche systématiquement le rôle d'intermédiaire social, symbolique, politique, rituel... Est-il alors légitime de les dénommer en français «artisans " s'ils ne pratiquent pas de métier? Mon propos n'est pas de remettre en cause cette traduction, mais plutôt de l'utiliser comme un point de départ pour m'interroger sur les implications de l'exercice d'une technique dans la définition de l'appartenance des inaden. Est-ce en sa qualité d'homme de métier que l'énad ${ }^{2}$ peut avoir des fonctions dans les domaines social, politique, rituel ou est-ce parce qu'il joue un rôle social et symbolique qu'il peut aussi être un homme de métier? Lexercice d'une technique le qualifie-t-il ou le disqualifie-t-il pour sa fonction sociale ? L'appartenance à cette catégorie sociale se définit-elle par l'activité, technique ou sociale, pratiquée ? Quelles conséquences l'exercice d'une technique a-t-elle dans la définition de leur statut social?

Cette série de questions oblige à s'intéresser à la place et à la valeur que les Touaregs attribuent aux techniques dans leurs systèmes de représentations et aux conséquences de cette pratique dans leur mode d'organisation 1996b : 228 ; Nicolas 1951 : 767 ; Rasmussen 1992 : 106 ; Saenz 1991 : 1 ; Norris 1975: 4.

2. Énad est le masculin singulier d'inaden. Le féminin singulier est ténadt et le féminin pluriel tinaden. 
sociale. À la suite de Pierre Lemonnier (1991: 697) et de Pierre Bonte (1985 : 20) notamment, il me semble que les techniques forment entre elles un système en relation avec d'autres systèmes de représentations (sociales, rituelles, symboliques...). Je vais ici tenter de montrer comment, du point de vue touareg, les faits sociaux et les faits techniques s'articulent (à partir de l'exemple de la fabrication) et dans quelle mesure les techniques peuvent avoir une fonction sociale et symbolique.

Pour cela, je me propose d'analyser plusieurs récits oraux en mettant en relief leur valeur de stéréotypes et en établissant des correspondances avec les représentations liées aux techniques. Ces récits portent essentiellement sur le statut des inaden en fonction de leur origine, sur la construction des liens qui les unissent avec le reste de la société touarègue, et sur la place des techniques de fabrication dans un système global de représentations.

Le cadre général de mes enquêtes de terrain se situe dans la partie ouest du monde touareg (territoire de l'actuel Mali), dans deux ensembles de type confédéral (ittebelen): celui des Imédédaghen et celui des Iwellemmeden Kel Ekümmed ${ }^{3}$. Ils occupent trois aires géographiques mitoyennes : l'Arabanda (rive sud du fleuve Niger), l'Awza (rive nord du fleuve Niger) et l'Azawagh (zone de plaines situées à l'est de l'Awza). Dans chacune de ces deux confédérations, différents lignages d'inaden vivent et parfois travaillent aux côtés de nobles (imushagh)t ou de tributaires (imghad) forts.

\section{Mythes: les enjeux des origines}

Marcel Detienne (1981) a montré, à partir de l'exemple grec notamment, que la fonction des mythes n'est pas d'expliquer le passé mais bien le présent. Il souligne par ailleurs qu'ils sont l'objet d'ajustements en fonction des contextes. Dans cette perspective, l'analyse des légendes et des mythes que

3. La confédération des Imédédaghen est un vaste ensemble politique occupant une zone discontinue allant de l'Ayr (Kel Tamgak) à l'Azawad (Kel Ehan Daw Ishkan). La matrice de cet ensemble est la fraction des Imédédaghen Kel Adagh, résidant actuellement dans l'Arabanda. Les Iwellemmeden Kel Ekümmed forment un ensemble politique composé notamment des fractions Kel Télateyt (ou Kel Tamolaqist), Kel Ahara, Kel Tagiwalt, Ibawen... Nous verrons par la suite les liens qui unissent ces deux ensembles politiques.

4. D’après Hélène Claudot-Hawad et Mahmoudan Hawad (1987: 554) imushagh, ou imajeghen selon les parlers, désigne au sens restreint les Touaregs nobles, et au sens large les Touaregs. Selon André Bourgeot (1993: 50), "...trois mots: mujegh (être touareg noble), emajagh (aristocrate guerrier) et temacheq (la langue touarègue), satellites de la même racine (MJGH), renvoient tous à l'aristocratie ». D'après Dominique Casajus "amajegh a deux sens dont l'un dénote, grosso modo, une appartenance ethnique ou linguistique, l'autre un statut social» (1987a : 23-24). Il note : "... une racine MJGH (ou $\mathrm{MJH}$, ou MZGH, ou MSGH) sert à désigner plusieurs populations berbères, et certains auteurs ont proposé, non sans vraisemblance, de la rapprocher du mot mazix (pl. mazices) par lesquels les Anciens ont parfois désigné $[. .$.$] certaines populations puniques de l'Afrique du Nord... ».$ 
j'entreprends ici, n'a d'autres fins que de comprendre la situation actuelle des membres de la catégorie sociale des inaden. Les récits à caractère mythologique où ils interviennent ont une valeur structurante. Pour la plupart, ils constituent un corpus nouveau, qu'il m'a été donné de recueillir dans de nombreuses régions du monde touareg et auprès de catégories sociales variées (nobles, tributaires, lettrés musulmans, "artisans", serviteurs...). Ces récits justifient, voire légitiment, le statut actuel des inaden et constituent un substrat sur lequel sont construites les relations entre ce groupe et le reste de la société. Dans chacun des récits, des enjeux différents se cristallisent autour de la question des origines. Dans quelle mesure le statut des inaden peut-il varier en fonction du mythe de référence?

\section{L'origine controversée des "artisans touaregs" (inaḍen)}

L'origine des inaden a suscité quantité d'hypothèses. Plusieurs auteurs évoquent une origine juive (Foucauld 1951-52, III : 1300 ; Nicolaisen 1962 : 71 ; Casajus 1987b : 294). Leur statut, jugé singulier par rapport aux autres groupes qui composent la société, découlerait de cette origine considérée comme exogène. Dans les deux confédérations de mon enquête, cette origine juive m’a également été énoncée ; le terme employé dans ces régions pour désigner les juifs est le terme arabe el yaoud, l'expression Banu Israël (litt. "Fils d'Israël ») n'est pratiquement jamais employée. L'origine juive des inaden est donc présente sous des formes proches au nord, à l'est et à l'ouest du monde touareg. Comment a-t-elle été construite et quelle conséquence a-t-elle sur le statut des inaden?

Juifs versus Arabes

Dans les lieux de mon enquête, ce sont essentiellement ceux n'appartenant pas à la catégorie sociale des inaden qui leur assignent cette origine et, d'après leurs dires, de nombreux indices en seraient la preuve.

Leur origine géographique est souvent présentée comme un argument majeur. Certains inaden affirment, en effet, venir du Sousse, de l'Anti-Atlas marocain (Tafilalet) ou du Touat, régions où vivaient respectivement des groupes berbères judaïsés (Jemma 1971 : 25; Schroeter 1997) ainsi qu'une importante communauté d'artisans juifs (Oliel 1994: 53). De nombreux personnages mythiques touaregs sont également considérés comme originaires de la Tafilalet, pour autant qu’il soit systématiquement fait référence à une appartenance à la communauté juive. Ainsi, d'après des récits oraux relevés dans l'Arabanda, Tin Hinan, ancêtre fondateur et matrice de la confédération des Kel Ahaggar, serait originaire de la Tafilalet (Norris 1972 : 85). Là, elle aurait trouvé des inaden qu'elle aurait pris sous sa protection. Danièle Jemma (1972), s'interrogeant sur la fonction sociale du groupe des inaden de 
l'Ahaggar, évoque également la question de leurs origines. Dans ce cas précis, ils viendraient de l'Ayr ou auraient été razziés par erreur dans l'Adghagh des Ifoghas (ibid. : 270). Par ailleurs, Henri Lhote (1984: 173) note que : «Selon les traditions, les Kel Ahaggar n'auraient pas eu de forgerons avant le sultan Goma à qui le sultan du Maroc en aurait offert ». L'auteur précise ensuite que des inaden furent razziés par les Kel Ahaggar chez les Kountas Dag ech cheikh dont certains seraient des descendants des juifs de Tamentit.

En se fondant sur cette origine géographique et par association, les inaden, parce que majoritairement artisans, sont considérés comme des juifs. Leur pratique de la bijouterie serait là, dit-on, pour en témoigner. Ce stéréotype associant l'origine à la fonction repose, me semble-t-il, sur le fait que dans de nombreuses régions du pourtour méditerranéen, la bijouterie, la fonderie et la ferblanterie sont le monopole d'une diaspora juive (Algérie, Tunisie, mais aussi Yémen...), alors que les artisans exerçant la forge sont plutôt de confession musulmane (Agabi 1997 : 2890). Dans les représentations populaires de l'Ouest du Sahara, relevées par les géographes arabes médiévaux et toujours vivaces, les Berbères sont présentés de façon stéréotypée comme des éleveurs de chèvres, les juifs comme des artisans (Norris 1972: 80). Si certains d'entre eux, comme ceux de la Tafilalet, sont de confession juive, rien ne nous dit s'ils sont des Berbères judaïsés ou des juifs berbérisés et rien ne prouve que tous les artisans du nord au sud et de l'est à l'ouest du Sahara sont juifs, même s'ils exercent la bijouterie.

Dans le même sens, Tal Tamari (1997: 10 et 85) indique que les voyageurs portugais du XVI ${ }^{\mathrm{e}}$ siècle employaient le terme " juifs " pour désigner les artisans de l'Afrique occidentale. D'après l'auteur (ibid. : 10), l'usage de ce terme :

" ... reflète évidemment les structures sociales de l'Europe à leur époque; ils étaient persuadés que des personnes soumises à une forte ségrégation sociale ne pouvaient avoir d'autre ascendance que juive et ils indiquaient par ailleurs que les "juifs" de l'Afrique occidentale avaient les mêmes pratiques religieuses que "les autres noirs"..." ".

Elle souligne également que le terme "juif» a ensuite été remplacé par celui de "caste» (ibid.). Cette appellation, issue du portugais casta, dont le sens premier est " race et espèce ", s'applique aux animaux comme aux hommes. Instaurée par les voyageurs de cette époque, elle perdure encore et semble avoir eu la même fortune que l'appellation "juif». L'une et l'autre n'ont été introduites qu'à la suite d'une perception superficielle de la condition des gens de métier ${ }^{5}$. type d'appréhension théorique qui présente tous les inconvénients du nominalisme. On caractérise .../... 
Ce stéréotype de l'origine juive est également repris par les lettrés musulmans (kel esuk et ishériffen), pour lesquels, en accord avec l'islam, il ne peut y avoir d'origine humaine que prophétique. D'après leurs dires, les inaden, dont certains exercent les activités de forge et plus rarement celle de fonderie (Hincker 1996), descendraient du prophète (anebi) Daoud qui avait le pouvoir de transformer les pierres en métal. D'autres prétendent au contraire qu'ils descendent de son fils, le prophète Souleyman, qui commandait aux vents et qui leur aurait inspiré le soufflet ${ }^{6}$. Dans ce stéréotype, l'accent est mis sur l'origine prophétique beaucoup plus que sur l'appartenance à la communauté juive de ces deux prophètes. En outre, l'origine juive de Daoud et Souleyman semble secondaire au regard de l'importance des découvertes techniques transmises aux inaden.

Rappelons que, dans l'islam, le statut des juifs n'est pas constant. Dans la première période de la prédication mohamédienne, qui fait référence à l'Arabie ancienne, les «Fils d'Israël » dans leur ensemble, et dont font partie Daoud et Souleyman, sont présentés comme vivant en harmonie avec les Arabes ${ }^{7}$. Par la suite, après l'arrivée de Mohamed à Médine (an I de l'Hégire, 622 ap. J.-C.), lors de la seconde période de prédication, l'harmonie entre les Sémites de la péninsule arabique est rompue et les "Fils d'Israël » rejoignent les chrétiens sous l'appellation d' «hommes des écritures ${ }^{8}$. Leur statut est désormais assimilé à celui d'apostats (kouffar).

Ces stéréotypes de l'origine juive ont tous en commun de mettre le métier qu'exercent certains inaden au centre de la définition de leur appartenance. Ils ne sont cependant pas associés aux seuls inaden.

De l'origine "juive" des Idaw Ishak ou Idaw Isahak

Selon certaines sources orales relevées dans l'Azawagh, les Idaw Ishak ou Idaw Isahak ${ }^{9}$ seraient également d'origine juive. Jacob Oliel (1994: 123) évoque l'existence d'un groupe, qu'il appelle «Daggatoun» (litt. "les descendants d'Atoun» ou de "Gatoun») et qu'il rapproche des Idaw Isahak essentiellement parce qu'ils seraient de confession juive et qu'ils vivent aux côtés des Iwellemmeden. Pour sa part, Charles Monteil (1951 : 292), citant le Rabbin Abi Serôr Mardochée, précise que les Daggatoun seraient des pas-

\footnotetext{
les groupes sociaux par un terme en fonction de leur conformité à un certain nombre de critères qui, conventionnellement groupés, sont constitutifs de la définition de ce terme. Autrement dit, le concept n'est porteur d'aucune vérité que la conformité à sa propre définition. "

6. Ces origines prophétiques reprennent la sourate XXXIV du Coran, dite de "Saba" (versets 10 et 11). 7. Voir par exemple la sourate X du Coran dite de "Jonas» (versets 90 et 93) ou la sourate XLV, dite de "la génuflexion" (versets 15 et 16).

8. Voir par exemple la sourate $\mathrm{V}$, dite de «la table» (verset 70 ).

9. Le terme «Idaw » est une particule qui désigne, dans l'Anti-Atlas marocain, certains groupes territoriaux. Sur ce point voir El Alaoui (2001 : 191). Par ailleurs, le terme Idaw Isahak semble être une transformation récente de l'appellation Idaw Ishak.
} 
teurs vivant entre la Tafilalet et Tombouctou, qu'ils seraient sous la protection des Touaregs et se battraient "au premier rang pour eux ». Outre leur origine juive, les Idaw Isahak posséderaient également une langue particulière, résultat d'un amalgame entre plusieurs idiomes: «Lorsque Dieu répartit les langues sur la terre, les Idaw Isahak dormaient et, arrivés en retard à la distribution, ils n'eurent que des restes de langue " (Awza, 1999) ${ }^{10}$.

Sous des formes diverses, cette légende est présente dans de nombreux points du monde musulman, de l'Inde à l'océan Atlantique. Loin d'être extraordinaire, la langue que parlent les Idaw Isahak est un dialecte zarma de Tombouctou parfois émaillé de quelques mots berbères. Ils forment un groupe de tributaires (imghad) des Iwellemmeden, instruits dans l'islam, qui aurait quitté, il y a fort longtemps, le sud de l'Ahaggar pour s'installer dans la région de Tombouctou en pays zarma ${ }^{11}$.

Harry T. Norris (1972: 46) a montré que de nombreuses légendes circulent d'une culture à une autre et sur des distances considérables. Certains récits populaires juifs ont influencé des traditions orientales orales; ils ont ensuite été importés en Espagne depuis le Yémen au moment de la présence almoravide. Dans le même ouvrage (ibid. : 64-65), l'auteur remarque qu'après les conquêtes arabes, les écrits des géographes (dont El Bekri) normalisent la tradition populaire en fonction des stéréotypes de l'islam. Ainsi, à propos de l'histoire d'Abdullah ibn Tifawt al-Lamtuni, qui créa une confédération rassemblant les Lamtuna, mais aussi les Gudala et les Banu Warith, et qui entra en conflit avec des populations noires judaïsées (les Bafur), l'auteur note (ibid.):

«But these negroes had something peculiar about them. "They professed Judaism”. This brief remark is significant. It must be seen in connection with the strange ideas of the geography and of the peoples of the Sahara entertained by the medieval Arab geographers. The world of fancy exists where the world of reality no longer conforms to the limited knowledge of the writer. Had not al-Hamadhani centuries earlier reported the presence of these Jews in a city in the furthest Sahara? Since then al-Idrisi and others had reasserted their presence and the afflictions which they had endured at the hands of the Lamtuna. Memories of these reports were to survive in Mauritanian folktales of the Bafur, the "judaized negroes", and others whose portrayal is distinguishable from those of dog-faced men and long-eared dwarfs, only in degree and not in kind.»

Comme l'a montré Gabriel Camps (1991 : 1395), le terme Bavares, mentionné dans des dédicaces des $\mathrm{III}^{\mathrm{e}}$ et $\mathrm{V}^{\mathrm{e}}$ siècles, désigne deux groupes distincts (d'une part, les Orientaux sur les Babors et, d'autre part, les Occidentaux en

10. Tous les récits oraux ont été recueillis en langue touarègue. Entre parenthèse figurent la région où le récit a été collecté et l'année.

11. Ces informations ont été recueillies dans l'Arabanda et dans l'Azawagh en 2000. Sans que leurs origines soient communes, il existe également un autre groupe touareg, appelé Elkaseybaten, vivant dans le sud de l'Arabanda et ne parlant aujourd'hui que la langue songhay. 
Maurétanie césarienne) rebelles à la soumission romaine. Dans le même texte (ibid. : 1399), il cite T. Lewicki (1978) qui fait un rapprochement entre les Bavares et les Bafour ou Bavur qui vivaient dans l'Adrar mauritanien au Moyen Âge. Par ailleurs, Pierre Bonte (1998 : 102) souligne que l'appellation Bavur est souvent associée à celle de "païens ". D'après ces différents auteurs, les Bafour appartiendraient ainsi aux catégories de l'altérité.

La présence juive dans de nombreuses régions du Sahara - dont l'Adghagh, par laquelle ont transité de nombreux groupes touaregs de l'ouest - à la période médiévale a été démontrée depuis longtemps (Monteil 1951 ; Camps 1982). Il semble donc possible que, par contacts, certains juifs aient pu être intégrés à des groupes berbères ou que, selon Camps, certains Berbères aient pu être judaïsés. La mention ou l'occultation de juifs dans la parenté varie selon les groupes touaregs et selon les enjeux que cette présence recouvre. Certains Touaregs ne cachent pas la présence d'ascendants juifs, comme d'autres d'ascendants peuls, songhay ou maures dans leur parenté (Badi 2002 : 56-57). Dans cette perspective, il convient donc de s'intéresser beaucoup plus aux représentations que recouvre la filiation juive qu’à son éventuelle authenticité.

L'essor de l'islam au Sahara a notamment propagé de nouveaux stéréotypes. Les juifs, du point de vue des musulmans, incarnent l'altérité absolue, celui qui n'est pas soi. Assigner à autrui une origine juive, revient à se démarquer franchement du proche ou du semblable. Aussi, dans l'ouest du monde touareg, les inaden n'évoquent-ils pas cette origine. Seuls certains nobles, tributaires ou lettrés musulmans, leur assignent une ascendance juive pour souligner la différence de statut qui les en sépare. Empruntée aux catégories musulmanes de l'altérité, l'origine juive met en évidence une distinction, comme pourraient l'être d'autres stéréotypes (caractéristiques physiques, coutumes alimentaires, vestimentaires...) ${ }^{12}$.

Intérieur versus extérieur

Dans le cas des inaden, comme dans celui des Idaw Isahak, le stéréotype de l'origine juive servirait à souligner que ces deux groupes seraient initialement extérieurs à la société touarègue et qu'ils auraient été, au fil de l'histoire, intégrés à celle-ci. En souvenir de leur origine exogène, leurs membres seraient situés aux marges de la société et auraient un statut particulier.

Ce schéma contredit en bien des points la réalité. Les exemples d'intégrations d'entités extérieures à la société touarègue ne manquent pas et les groupes, dont il n'est d'ailleurs pas toujours gardé la mémoire de leurs 
origines étrangères, ne bénéficient pas d'un statut particulier. Hélène Claudot-Hawad (1998) émet d'ailleurs l'hypothèse que seuls les tributaires sont considérés comme véritablement d'origine locale. Quantité de groupes nobles appartenant à l'élite de la société sont reconnus comme d'origine extérieure.

Ainsi les esclaves (iklan), qui jadis étaient le plus souvent razziés dans les groupes sahéliens voisins ${ }^{13}$, pouvaient être affranchis et devenir membres à part entière de la société lorsqu'ils se comportaient noblement (iknen tamujegha), c'est-à-dire lorsqu'ils appliquaient les valeurs qui régissaient les principes de l'appartenance touarègue.

Quantité d'appellations désignant des catégories sociales soulignent qu'elles résultent de "métissages " soit entre plusieurs catégories à l'intérieur même de la société, soit entre l'intérieur et l'extérieur. Ainsi, le terme irréganaten désigne-t-il un groupe de tributaires (imghad) ou de nobles (imushagh) formé de lignages d'origine différente. Le terme iboghelliten, désigne dans l'ouest un groupe de condition libre (illelen) composé de gens d'origine servile et de condition libre (Songhay ou Touaregs) ${ }^{14}$.

La question de l'origine de ces entités extérieures est primordiale et en même temps secondaire. D'une part, il est extrêmement valorisé de vouloir intégrer la société touarègue et, d'autre part, théoriquement, aucun être humain extérieur n'est plus apte qu'un autre à devenir membre de celle-ci.

Tisser des liens étroits avec des groupes libres voisins est parfois plus qu'une possibilité, une nécessité. Les Touaregs utilisent ainsi la métaphore des métaux précieux qui, pour être travaillés, doivent être mélangés à de petites quantités de cuivre. La société, comparée ici à l'or ou l'argent, ne peut fonctionner que si elle intègre des éléments extérieurs ${ }^{15}$. Certains mythes d'origine, relevés chez les Imédédaghen, racontent comment la fondatrice mythique de la fraction s'est engagée, lors de son arrivée dans l'Arabanda, à protéger des groupes peuls libres. Ces groupes peuls, aujourd'hui appelés ifulanen ${ }^{16}$, sont considérés comme des membres à part entière de la société touarègue ${ }^{17}$. Ils dépendent essentiellement de la confédération des Imédédaghen, ont adopté la langue berbère et certains traits culturels

13. Chez les Imédédaghen Kel Gossi, ils étaient par exemple razziés chez les Dogons de la plaine.

14. Pour plus de détails sur les conditions de l'affranchissement et du métissage, voir Claudot-Hawad (2000).

15. "Nous sommes du métal parfaitement noble, mais sans alliage nous ne pouvons tenir debout " (Claudot-Hawad 1996a : 8).

16. Il me semble que la construction même du terme ifulanen évoque leur statut. Ifulan est le mot qui désigne, en langue touarègue, les Peuls (Djelgodjé, Gabero, Bororo...). Les ifulanen sont, quant à eux, devenus dépendants au plan statutaire et c'est peut-être la raison pour laquelle leur appellation a pris la marque touarègue syntaxique du pluriel (-en).

17. Comme ils sont d'origine libre, il n'est pas attendu d'eux qu'ils acquièrent toutes les valeurs conditionnant l'appartenance à la société. Ils conservent leur mode de vie propre tout en résidant aux côtés des Touaregs. Ils sont associés au statut des dépendants (tilaqiwen). 
touaregs tout en conservant des signes extérieurs évidents de leur origine exogène (vêtement, habitat...). Par ailleurs, les Imédédaghen, comme de nombreux groupes de tributaires (Bernus 1983 : 134), se considèrent comme des descendants de Koseila, roi berbère qui tua le prédicateur musulman Oqba ibn Nafé. Il est peu probable que cette filiation soit réelle, mais se réclamer de ce personnage en dit long sur les rapports politiques qu'entendent entretenir les tributaires avec les Arabes ou les Berbères arabophones (Claudot-Hawad 1985).

En somme, le stéréotype de l'origine juive assignée aux inaden joue comme un processus de démarcation de cette catégorie sociale vis-à-vis du reste de la société. La pratique d'un métier apparaît ici bien secondaire par rapport à ce que recouvre l'assignation de cette identité.

\section{Ta Mellet ou Tamenannat}

Dans d'autres types de récits, l'origine des inaden se construit sur des bases et de manière différentes. Dans les confédérations imédédaghen et iwellemmeden, les nombreuses légendes que j'ai pu relever distinguent deux origines dans les lignages d'inaden qui leur sont associés. Il y aurait ceux qui descendraient de la branche de Tamenannat et ceux qui auraient pour origine celle de Ta Mellet ${ }^{18}$.

De nombreuses sources bibliographiques évoquent l'appartenance de certains inaden au groupe de Tamenannat ${ }^{19}$. Dans les représentations associées à l'étymologie de ce terme, il désignerait «tourner" ou "tordre ${ }^{20}$; il renverrait à la parole des inaden, qui sont passés maîtres dans l'art de tourner les mots selon les contextes et les situations pour parvenir à persuader leur auditoire. Outre cette capacité à emporter l'adhésion, à convaincre par les ruses de la parole, les membres de Tamenannat seraient également les spécialistes de la fabrication de la selle de cheval (élakef). Les femmes seraient les spécialistes du travail du cuir.

D'autres inaden revendiquent, par contre, une filiation au lignage dit de Ta Mellet. Ta Mellet (litt. : «la blanche») est un lieu situé sur les bords de la vallée du Tilemsi à l'est de l'Adghagh. Avant la destruction de la ville de Tademekkat, située dans cette même région, et où vivait la confédération

18. C. Saenz (1991 : 77) relève que, dans l'Ayr, il y aurait deux groupes d'inaden, ceux de Tamenannat et ceux dit Isedregen. Il note que le groupe des Itabeytabayen seraient des inaden de l'Ayr qui auraient migré vers l'ouest en suivant les nobles auxquels ils sont attachés (ibid. : 29).

19. Voir, par exemple, Saenz 1991: 77 ; Bernus 1983: 240 ; Benhazera 1908 : 72 ; Nicolas 1951: 768. C. Saenz $(1980: 6 ; 1991: 14)$ note que le terme tamenannat serait le nom d'une artisane qui aurait accompagné les nobles Kel Ferwan au cours de leur installation dans l'Ayr. C'est elle qui aurait donné naissance au groupe des artisans de l'Ayr et serait donc son éponyme.

20. Alojaly (1980: 140) note comme sens de la racine /NND/: «enrouler ", « envelopper "; « tourner dans une autre direction »; «être enroulé »; « être tourné ». 
imédédaghen ainsi que de nombreux groupes nobles (Igawdaren, irréganaten...) occupant actuellement les bords du fleuve Niger, des inaden résidaient et travaillaient. Dans les sources que j'ai recueillies, rien n'indique qu'ils exerçaient à cette époque le rôle d'intermédiaire sur le plan social et rituel. Par contre la majorité d'entre eux était détentrice d'un métier (forge et boissellerie pour les hommes, peausserie pour les femmes).

Une légende dit :

"Un jour les inaden de Tamenannat se sont rendus chez ceux de Ta Mellet. Chacun a alors cherché à affirmer la supériorité de ses richesses. Durant la nuit, les inaden de Ta Mellet ont trait leur bétail et ont rempli de lait une mare sèche en cette saison. À leur réveil, ceux de Tamenannat ne purent que constater la supériorité de la richesse des inaden de Ta Mellet" (Arabanda, 1999).

En tenant compte des règles qui régissent les rapports sociaux touaregs, d'après cet épisode il semblerait que ces deux groupes d'inaden sont égaux sur le plan statutaire puisqu'ils entretiennent des rapports de type compétitifs. Cela ne signifie évidemment pas qu'il en a toujours été ainsi et certaines autres légendes font du groupe de Ta Mellet une branche cadette de celui de Tamenannat. Actuellement, ces deux groupes forment un ensemble apolitique de lignages très étroitement imbriqués.

Cette rivalité entre deux lignages sur la base d'une différence d'origine a également été relevée par Danièle Jemma (1972 : 270) dans l'Ahaggar :

«Les descendants d'Alamin, quant à eux, purent conquérir la suprématie sur les descendants de Sama parce qu'ils furent amenés en Ahaggar par les Kel-ghela, à l'époque de l'établissement de l'hégémonie de ces derniers sur les Touaregs de l'Ahaggar qui dépendaient antérieurement du Sultanat des Immenan. »

Chez les Imédédaghen, j'ai relevé que les descendants d'une ténadt, dénommée Fawa, seraient plus forts statutairement que les descendants d'une autre ténadt appelée Tadetosset. Fawa et Tadetosset dépendent respectivement de deux fractions de la confédération imédédaghen (Kel Gossi et Kel Ehan Ishagaghen).

À une autre époque, au milieu du XX $x^{\mathrm{e}}$ siècle, un conflit opposa les inaden des Imédédaghen Kel Gossi à ceux des irréganaten ${ }^{21}$. Au cours d'une rixe, un énad des irréganaten tua un énad des Imédédaghen Kel Gossi. La crise qui s'ensuivit dégénéra rapidement, les hommes se préparèrent au combat, jusqu'à ce qu'un énad de renom (autrement dit un amghar) rappelle aux protagonistes l'imbrication de leur parenté et l'équivalence de leurs forces. Ces propos suffirent à calmer les tensions.

21. Les irréganaten de Gossi sont des tributaires (imghad) de trois branches différentes: Kel Bakaten, Idermagen, Ibergan. 
On constate, au travers de l'analyse de ces légendes, que, d'une part, la catégorie sociale des inaden est composée de différents lignages, peut-être initialement de statuts différents et, d'autre part, que la pratique d'un métier ne semble pas constituer un enjeu dans leur hiérarchisation.

\section{Une structure mythique commune}

Une autre série de récits stéréotypés, mettant cette fois en scène les inaden et les groupes dont ils dépendent, souligne l'association primordiale entre ces deux groupes. Ces mythes associent un groupe d'inaden aux Imédédaghen, au moment de leur réorganisation politique dans les territoires du sud (Arabanda), après la destruction de la ville de Tademekkat ${ }^{22}$. Dans cet ensemble de type confédéral comme dans d'autres, l'ancêtre mythique est une femme qui vient du nord escortée de ses filles (Claudot-Hawad 1993: 126). Takhata, l'ancêtre mythique des Imédédaghen Kel Gossi, lorsqu'elle pénétra dans l'Arabanda, était accompagnée d'une ténadt:

" [En ce temps là, les Imédédaghen résidaient dans l'Awza et partaient régulièrement à Hombori, dans l'Arabanda, dont ils convoitaient le contrôle, $N d t$. Quand ils sont revenus [dans l'Awza], ils ont dit à leur chef: "Nous avons vu des faibles (tilaqiwen) qui possèdent des biens. Ces faibles ne sont couchés sur personne et tout le monde peut les piller." C'est alors que Takhata s'est levée, elle a dit : "J'y vais." Elle avait des filles, je ne sais plus si c'est deux ou cinq. Elle est partie chez un noble (amashagh) de ceux des Iwellemmeden. Elle a demandé à avoir un énad. Elle a dit: "Je vais dans une région que l'on nomme Gossi, je viens pour avoir une ténadt." Il lui a donné Fawa qui était enceinte et avait un jeune garçon. C'est cette ténadt qui lui a cousu des outres. Il lui a aussi donné une captive (taklit). Ensemble elles ont déménagé. Ensemble elles se sont installées à Aghey [au nord-est de Gossi, $N d t$ et c'est là que Fawa a accouché " (Arabanda, 1998).

Dans ce mythe, dont existent plusieurs versions, Takhata la fondatrice part dans l'Arabanda pour créer un nouvel ensemble politique en s'alliant avec un groupe d'hommes riches sur le plan matériel mais faibles sur le plan politique (tilaqiwen). Il s'agit de la catégorie sociale des irréganaten. Le mythe souligne que la création de cette nouvelle fraction s'accompagne d'une mise en place des différents corps qui composent la société (catégories sociales élevées - ici tributaires, imghad-, des inaden et gens de condi-

22. La ville de Tademekkat, également appelée esuk, située dans l'Adghagh sur la route de Ouargla était jusqu'au XV $\mathrm{XV}^{\mathrm{e}}$ siècle un très important centre religieux. Les causes de sa destruction sont l'objet d'un débat. La thèse la plus largement admise par mes interlocuteurs, et également reconnue par les Ifoghas comme l'a montré Dida Badi (2002), serait l'assèchement des points d'eau environnants la ville. Selon d'autres sources, la ville aurait été détruite par les Iwellemmeden pour asseoir leur hégémonie sur l'Adghagh. Enfin, une autre source largement inspirée d'El Bekri (1913) imputerait sa destruction aux Songhay, par ailleurs fondateurs de cette ville selon cette même source. Sur ce point, voir Norris 1975 . 
tion servile, iklan). Takhata est présentée comme la matrice de cette nouvelle unité politique. Fawa, la ténadt qui l'accompagne, aura comme fonction de lui fabriquer des objets en cuir $^{23}$.

Dans ce mythe, les conditions sociales requises pour qu'une fraction voit le jour sont exposées. Son existence semble bien dépendre non pas de la seule matrice (ici une tributaire), mais de l'existence de deux couples : femme-noble/ténadt et femme-noble/taklit. Il est ainsi mis en évidence que la première condition, lorsque Takhata se décide à partir à Gossi pour former une nouvelle tribu, est de s'associer à une ténadt.

Ce mythe donne également des informations sur les relations qu'entretiennent les descendants de Takhata avec les nobles (imushagh). Les Imédédaghen, quelle que soit la fraction à laquelle ils appartiennent, sont considérés comme des tributaires (imghad) forts. Ils bénéficient actuellement d'une grande autonomie politique par rapport à d'autres groupes tributaires, mais il n'en a probablement pas toujours été ainsi. Ils ont été, selon les périodes de leur histoire, des alliés ou des dépendants de nobles Iwellemmeden. Imédédaghen signifierait, selon toute vraisemblance, " ceux de l'Adagh ». Avant la destruction de Tademekkat, ils étaient déjà, semble-t-il, à la tête d'un ensemble politique de type confédéral (ettebel). Le fait que Takhata demande à un noble (amashagh) de lui confier une ténadt pourrait souligner que ce mythe renvoie à un passé situé après la destruction de Tademekkat, lorsque les Imédédaghen dépendaient statutairement des Iwellemmeden, mythe qui, en outre, expose comment la fraction des Kel Gossi s'est détachée de sa propre matrice (les Imédédaghen Kel Adagh) pour former une fraction autonome.

À partir de ce récit, j'envisage plusieurs hypothèses. Tout d'abord, il semble que les Imédédaghen Kel Gossi n'avaient pas d'inaden au moment de la création de leur fraction. Alors que leur matrice (les Imédédaghen Kel Adagh) en avait lorsqu'elle résidait aux environs de Tademekkat. Il est envisageable que leurs inaden aient rejoint le groupe des Iwellemmeden après leur affaiblissement. Ensuite, les légendes relatives au groupe de Ta Mellet, originaire de l'Adghagh, ne précisent pas si ce groupe vivait là avant ou après la destruction de Tademekkat et s'il dépendait d'une confédération particulière.

D'après les propos que j'ai recueillis, Fawa, qui savait travailler le cuir, serait issue du groupe de Tamennanat, et la preuve en serait qu'elle était, au moment du départ de Takhata, aux côtés des imushagh Iwellemmeden. Les inaden issus du groupe de Tamennanat sont, en effet, constamment associés aux nobles.

23. Pour plus de détails sur les représentations associées au travail du cuir, voir Hincker 2002. 
En somme ce mythe, relatant la genèse d'une fraction, souligne l'étroite association entre les groupes politiquement forts, qu'ils aient le statut de noble (imushagh) ou de tributaire (imghad), et d'un ou plusieurs lignages d'inaden. Il montre que le groupe des tributaires (imghad) et celui des inaden répondent à la même structure mythologique : une femme matrice, qui possède déjà une descendance, crée un nouveau lignage à partir de l'extension d'un lignage déjà existant (Claudot-Hawad 1993 : 68-72). D'après ce mythe, rien ne distingue en apparence les coutumes matrimoniales des tributaires de celle des inaden, si ce n'est le genre de la descendance qui va se marier sur place. Takhata marie ses filles, alors que Fawa marie son fils. Ce point souligne peut-être la prévalence, pour les inaden, de l'agnatique sur le cognatique. Rappelons ici que, dans le cas des tributaires (imghad) de l'Ouest, les règles de descendance suivent le plus souvent la ligne utérine, alors que dans le cas des inaden, le principe d'unifiliation n'est pas présenté comme la règle. Les uns comme les autres constituent un groupe de descendance, mais les inaden ne forment pas de tawsit (fraction ou tribu), pour des raisons vraisemblablement liées à leur statut ${ }^{24}$.

Il ressort de l'analyse de cette première série de récits oraux que la question de l'origine des inaden est étroitement liée à leur statut au sein de l'édifice social touareg. Tout en utilisant des arguments divers, l'ensemble de ces récits a en commun d'insister sur la proximité et en même temps sur la différence qui existent entre les nobles, les tributaires, d'une part, et les inaden, de l'autre. Ils n'évoquent que très rarement l'enjeu que représente la pratique d'une activité technique.

\section{Différenciation sociale des rôles et des statuts}

La question qui va nous occuper dans ce paragraphe est de savoir si le rôle rituel et l'activité technique des inaden sont, d'un point de vue touareg, deux activités opposées ou complémentaires.

\section{De l'origine de la répartition des tâches}

Dans toutes les zones de mes enquêtes et en me référant aux ouvrages existant, il apparaît que la répartition des techniques de fabrication en fonction des genres est constante dans l'ensemble du monde touareg. Le

\footnotetext{
24. D’après André Bourgeot (1972: 536), «Les Enaden se caractérisent par une tendance à la dispersion ; leur campement regroupe une ou deux familles, ou bien encore s'intègre, tout en demeurant spatialement marginal dans la vie des campements suzerains ou vassaux ". D'après Dominique Casajus (1987a : 30), "Chaque forgeron est attaché avec les siens à une ou plusieurs familles de nobles ou de roturiers pour lesquels il est une sorte de client, il vit sur la terre de la tribu de ces patrons. [...] De ne pas être attaché à un territoire comme le sont les autres Touaregs donne aux forgerons une position particulière dans la société ».
} 
travail du métal et celui du bois sont des activités masculines, alors que le travail des peaux est essentiellement féminin. Deux récits recueillis dans l'Arabanda relatent que :

"Au commencement du monde, il n'y avait pas que les inaden, il y avait trois sortes de gens: les inaden, les nobles (imushagh) et les captifs (iklan). Au commencement de notre monde d'ici, une captive est partie, elle a pris son panier en vannerie, elle est allée chercher du fonio et elle en a trouvé. Une ténadt est partie et a cousu une outre, elle a cherché de quoi manger. [...] La ténadt a donné l'outre à la captive (taklit) et la ténadt a eu en échange du fonio " (Arabanda, 2000).

Ce récit montre que la fabrication des plats et paniers en palmier doum (Hyphaene thebaica) est, dans le groupe des Imédédaghen où il a été récolté, essentiellement l'activité des femmes d'origine servile ${ }^{25}$. Le plat évoqué ici est très certainement un panier à claire voie et fond plein, servant à récolter le fonio. Par contre, la fabrication de nattes brise-vent (eseber), de fines nattes de lits (tawsit) ou de certains types de couvercles (asahagh) en tiges de graminée sauvage (Panicum turgidum) sont fabriqués par les femmes appartenant à la catégorie sociale des tributaires (timghadt, fem. de imghad). La ténadt de ce récit, quant à elle, travaille les peaux, technique que ne détient visiblement pas la captive d'origine servile puisqu'elle échange du fonio contre une outre en peau tannée. Les activités techniques féminines semblent donc réparties en fonction de l'appartenance à telle ou telle catégorie sociale.

Une autre légende construite sur le même principe, insiste sur les conditions qui ont motivé la répartition sociale des tâches :

"On dit que les gens d'avant ont réparti les tâches. Ils ont donné le travail du bois et du métal aux inaden parce qu'ils n'étaient pas nombreux. Ils ont donné le travail de l'étude des Livres aux kel esuk, également parce qu'ils n'étaient pas nombreux. Ils ont donné les armes aux nobles parce qu'ils étaient les plus nombreux. [...] Et c'est ainsi que chacun a eu son propre caractère (tashni)» (Arabanda, 2000).

Au-delà de l'importance du nombre des individus avancé pour justifier la répartition des tâches, il est intéressant de signaler, que le travail du bois et du métal sont bien, dès les origines, des activités masculines réservées aux inaden. À chaque catégorie sociale est associée, de façon stéréotypée, une activité spécifique. Elle confere, dit-on, un caractère particulier aux individus qui la pratique. Le terme tashni (tazni ou tajné, selon les parlers) renvoie à un type de tempérament et de comportement propre à chaque

25. T. Benfoughal (1996: 58) note que l'utilisation de la matière "palmier dattier " (Phoenix dactylifera) est surtout réservée, chez les populations sahariennes vivant en Algérie, aux agriculteurs sédentaires. Il est aussi à signaler que les femmes du groupe des Izagayaten, résidant dans le nord de l'Arabanda, sont spécialisées dans la confection de nattes de lit (eserar) en palmier doum. Elles fabriquent également quelques coussins et petits sacs en cuir de bien moins bonne qualité que ceux faits par les tinaden. 
catégorie sociale ${ }^{26}$. D'après ce récit, l'activité pratiquée forge le caractère et celui des nobles ne ressemble pas à celui des inaden, car leurs activités sont différentes. L'implication des individus dans leur fonction est ici considérée comme primordiale et permanente au point que des traits spécifiques tendent à les différencier de ceux qui ne pratiquent pas les mêmes activités.

La question de l'ancienneté, dans la définition d'une identité de métier, est centrale pour les inaden. Celui qui n'a pas d'histoire (atarekh) dans le métier ne peut parvenir, dit-on, à l'apprendre (Casajus 1987b : 293). Elle joue également un rôle dans l'acquisition de compétences techniques. Celui dont les ascendants sont inaden (qu'ils pratiquent une activité technique ou non) détiendrait des prédispositions à l'apprentissage technique que ne possèdent pas les autres catégories sociales. Ces aptitudes particulières seraient transmises par le sang (ashni, azni ou ajnè). Cette représentation souligne l'importance de l'identité des ascendants dans l'acquisition des connaissances techniques. Dans cette perspective, les inaden se considèrent, et sont considérés, comme les légataires des techniques de fabrication.

\section{Activités sociales des inaḍen}

S'agissant de l'étymologie du terme énad, plusieurs hypothèses sont avancées. Dominique Casajus (1987a : 223) rattache ce terme au verbe äned, dont le sens est «être à part, n'être comparable à aucun autre ". L'étymologie, qui m'a été énoncée dans l'Arabanda, rapprocherait ce terme de la racine /ND/, donnant le verbe nadu qui signifie : "décider, ordonner, commander, régner, conseiller»(Alojaly 1980 : 140). Le nom verbal (tanadt, plur. tinaden) désigne «la décision, l'ordre, l'autorité, le pouvoir, le conseil ». L'énad serait donc, entre autres, un conseiller attaché au pouvoir et à l'autorité, bref aux instances qui décident. Cette étymologie a la valeur d'un stéréotype qui met l'accent sur les représentations associées à la fonction et au statut de l'énad.

Par ailleurs, le fait de se décharger d'une partie de son travail sur une autre personne se dit en langue touarègue tesinahad, son sens est proche de celui de "confier la responsabilité de quelque chose à quelqu'un ». L'énad serait donc celui à qui l'on confie une responsabilité. Sa fonction dépend étroitement de l'exercice du pouvoir et c'est sans doute la raison pour laquelle, il est essentiellement associé aux fractions fortes sur le plan politique. Selon les contextes, l'énad exerce le rôle de diplomate, d'émissaire, d'homme de confiance, il intervient dans les rites de passage ${ }^{27}$, s'occupe de la réception des étrangers... En résumé, il joue le rôle d’intermédiaire.

26. Ghubeyd ag Alojaly $(1980: 84)$ traduit le terme tajné par «la coutume, la bonne conduite ». D’après Dominique Casajus (1987a : 106) ce terme souligne la consanguinité par exemple entre un homme et le fils de sa sœur.

27. Dominique Casajus (1987a) détaille ainsi le rôle de l'énad dans les cérémonies de mariage. 
À la différence des tributaires (imghad) et des nobles (imushagh), l'énad n'est pas soumis au code de l'honneur et il est placé en dehors de la compétition qui régit les rapports égalitaires entre les individus (Rasmussen 1992 : 105 ; Bernus 1983). Il me semble que l'énad n'est ni un pair ni un inférieur par rapport aux autres catégories qui composent la société28. Hors de la compétition pour l'honneur, il bénéficie d'une liberté de parole. Prendre ombrage de ce que dit un énad reviendrait à le considérer comme un pair et donc introduire une relation d'égalité avec lui. Or, les relations entre les inaden et le reste de la société ne doivent rester ni hiérarchiques, ni égalitaires; c'est là l'une des conditions de l'existence de cette catégorie sociale ${ }^{29}$.

Dans cette perspective, il me semble qu'il serait abusif de croire que les inaden sont soumis aux nobles ou aux tributaires auprès desquels ils vivent. Ils sont de condition libre et peuvent décider par eux-mêmes de quitter un lignage lorsqu'ils sont en désaccord avec les membres de celuici. En arriver à cette extrémité jette un discrédit profond sur le lignage que les inaden quittent. L'homme d'honneur doit en effet, théoriquement, protéger ceux qui sont faibles statutairement et qui ne peuvent se défendre. Laisser partir un énad revient à admettre son incapacité à le défendre, à le nourrir... De la même manière, un homme d'honneur ne frappe pas un énad, non pas parce que c'est un faible, un inférieur... mais bien parce qu'il n'est justement ni un pair ni un inférieur.

L'analyse de cette deuxième série de textes, confrontée à des données ethnographiques, montrent que, statutairement, les inaden peuvent à la fois intervenir dans le domaine politique rituel, social et technique et que le cumul de ces deux types d'activités n'a rien d'incompatible. Il nous reste maintenant à établir les liens qui unissent ces deux catégories d'activités.

28. De nombreux auteurs ont insisté sur le rapport qui existe entre le statut des inaden et les interdits sociaux qu'il transgresse (verbaux et comportementaux). Du fait de ces transgressions, il est craint et méprisé (Bourgeot 1972: 536 ; Rasmussen 1992: 106; Casajus 1987a : 222-223 ; Saenz 1991: 85 120). Hélène Claudot-Hawad (1996b : 228) montre que ce statut est associé à une signification d'ordre cosmogonique. L'énad appartient au monde de l'entre-deux et les activités techniques et politiques qu'il assume sont reliées à ces origines.

29. D’après H. Claudot-Hawad (2000: 237), « ...l'organisation sociale des Touaregs s'appuie sur deux principes, apparemment contradictoires, d'égalité et de hiérarchie...». Un axe horizontal «structure sur le mode égalitaire, des entités collectives ou individuelles paires, de même statut, dont les interrelations sont de nature compétitive et de caractère ombrageux. [...] Un deuxième axe, vertical, ordonne hiérarchiquement les différentes catégories sociales dont les relations - qui se déroulent sur le mode de la plaisanterie codifiée - sont gérées par le supérieur statutaire...». Elle note par ailleurs (ibid. : 238) que "la coexistence de ces principes - égalitaires et hiérarchiques - au sein du même système semble facilitée par l'intervention d'un troisième trait, c'est-à-dire un principe dynamique qui organise la transgression des catégories propres aux deux premiers axes ». 


\section{La valeur sociale des techniques}

Les activités techniques et sociales pratiquées par les inaden sont au cœur de la définition de leur statut. Dans ce qui précède, nous avons vu que c'est au même moment mythique que les activités ont été réparties, et c'est une gestion globale de leurs spécificités et des qualités requises pour les mener, qui a été prise en compte. Aussi, les représentations associées aux techniques de fabrication doivent-elles être considérées globalement et dans les rapports qu'elles entretiennent avec d'autres systèmes de représentations (rituelles, politiques, mythologiques...). C'est à cette seule condition qu'il apparaît possible de comprendre pourquoi les inaden, plus qu'une autre catégorie sociale, sont les détenteurs de connaissances techniques mais aussi esthétiques spécifiques.

\section{Le couple noble/énaḍ}

Le couple noble/énad (ou tributaire/énad) se présente, dans bien des contextes, comme une entité à double visage dont chacune des composantes s'oppose et se complète. Les stéréotypes qui les caractérisent sont exactement inverses. Les nobles sont considérés comme des guerriers auxquels toute une série de valeurs positives est associée : le courage, la générosité, la beauté... Inversement, les inaden sont présentés comme peu courageux, enclins à la rapine... Dans le rituel de mariage, Dominique Casajus (1987b : 301) souligne ainsi que la danse des nobles (imajeghen Kel Ewey) est empreinte d'une grande élégance, alors que celle des inaden est grotesque. Edmond Bernus (1983 : 242) note que: "Dans la littérature, le forgeron touareg a toujours été la victime d'une image stéréotypée où les qualificatifs de rusé, lâche, menteur, méprisé, reviennent avec une étonnante constance. "

Ces stéréotypes renvoient à un univers métaphorique où il n'est, en fait, question que d'une seule personne : l'homme touareg, présenté dans toute sa complexité. Ce couple incarne le double aspect positif et négatif de la condition humaine, l'un n'ayant d'existence légitime que par rapport à l'autre.

Le couple noble/ énad n'est d'ailleurs pas le seul à refléter la dualité, voire la complexité de l'existence de l'homme. Cette entité rassemble deux individus de condition libre alors que le couple noble/akli met en évidence, également sur un mode ambivalent, l'opposition entre la liberté et la servilité. L'homme libre, de quelque catégorie sociale qu'il soit (noble : amajagh; tributaire: ameghid; lettré musulman : aneslim, aw esuk; artisan : énad...) s'oppose ainsi à l'homme de condition servile (akli). Là aussi, de nombreux stéréotypes les opposent tout en les rassemblant (ClaudotHawad 2000). 
Les rapports entre les nobles et les inaden, tout comme ceux qui régissent les relations entre les hommes libres et ceux d'origine servile, sont l'expression d'une conception particulière de la nature humaine. L'autre, n'étant qu'une facette de soi-même, il ne peut être ni déconsidéré ni méprisé, car le dédaigner reviendrait à se dédaigner soi-même.

Aussi, ces stéréotypes ont-ils une valeur essentiellement théorique et, dans la réalité, les rapports entre les nobles et les inaden sont-ils d'un tout autre ordre. Dans la plupart des cas observés, les nobles se contentent de dire, souvent sur le ton de la plaisanterie, qu'ils déconsidèrent les inaden en raison de leur couardise, des mensonges qu'ils proferent..., mais dans les faits, ils ne leur témoignent aucune mésestime. En outre, un noble n'associe jamais un énad en particulier à ces critères dévalorisants. Ils restent toujours généraux, énoncés sur le mode «les inaden sont ... » et, ne s'applique à aucune personne en particulier. C'est à ce titre que les qualificatifs qui leur sont associés sont des stéréotypes dont la fonction n'est pas de déprécier les inaden pour eux-mêmes.

Le noble est nécessaire à l'énad et l'énad est nécessaire au noble. Il ne faut donc considérer leurs rapports que sur un mode complémentaire. On ne peut juger qu'un énad bénéficie d'une grande liberté de parole que par rapport à la retenue dont fait preuve le noble ; l'énad n'est peureux que par rapport au courage du guerrier, il ne quémande que pour rappeler le noble à la générosité... Les rapports du couple noble/énad ne sont pas construits sur la négation d'une partie de soi, mais plutôt sur sa gestion et son acceptation. C'est sur cette base de complémentarité, que sont construites les relations sociales entre les individus selon un axe vertical de type hiérarchique, comme l'a souligné Hélène Claudot-Hawad (2000 : 237).

\section{Politique versus technique}

Si les nobles (imushagh) ou les tributaires (imghad), et les inaden sont deux aspects d'une même réalité, qu'en est-il de leurs activités ? Les nobles exercent, notamment, toutes les activités relevant du politique (exercice $\mathrm{du}$ pouvoir, gestion des ressources du territoire, guerre...); les inaden interviennent dans des activités rituelles, sociales et techniques. En suivant cette logique de l'association des contraires, il apparaît légitime de se demander si la technique est le pendant du politique.

S'agissant des nobles comme des inaden, force est de constater que l'un n'est pas rigoureusement exclu des activités de l'autre et réciproquement. Les femmes le plus souvent issues de la catégorie sociale des tributaires exercent quelques activités techniques, tannent les peaux, réalisent le vélum de tente et fabriquent des nattes en fibre végétale ${ }^{30}$. Les inaden, quant à eux, prennent part à certaines activités politiques notamment 
diplomatiques. Cependant, il est des domaines où l'un et l'autre sont nettement séparés. Les femmes tributaires, si elles épilent, tannent, coupent et cousent pour fabriquer une tente, et éventuellement de menus objets, ne détiennent pas de connaissances esthétiques aussi étendues que les inaden (Hincker 2002). Inversement, les inaden ne font pas partie de l'assemblée (tidawt) qui incarne le pouvoir politique, même s'ils jouent un rôle consultatif et exercent ainsi un pouvoir indirect ${ }^{31}$.

À la lumière de ces exemples, il semble que l'opposition statutaire entre les inaden et les nobles ne repose pas sur l'opposition entre le politique et les techniques. Ces deux groupes se distinguent essentiellement par la nature des activités qu'ils endossent dans ces deux domaines. La pratique d'une activité politique ne semble pas exclure de la pratique d'une activité technique et réciproquement. Chacune de ces deux catégories sociales, parce que de statut différent, manifeste un rapport distinct, et en même temps complémentaire, dans ces deux domaines.

\section{Statut social des inaden et valeur symbolique des techniques}

Comprendre le statut des inaden passe par une analyse de la valeur symbolique et du statut des techniques et des conceptions esthétiques.

Le personnage légendaire d'Amamellen est très connu à l'ouest comme à l'est, au nord comme au sud. Il intervient dans des moments cruciaux de l'organisation du monde ou de la société touarègue (Claudot-Hawad 1987 : 556-558; Casajus 1987a : 104, 283, 294).

Dans une légende connue dans tout le monde touareg, Amamellen démontre à sa femme l'utilité des inaden:

«La femme d'Amamellen se plaignait toujours de ce que les inaden réclamaient leur part [en nourriture, en habits...]. Un jour où son mari n'était pas là, elle les chassa. De retour, il lui demanda où ils étaient partis. "C'est moi qui les ai chassés!", répondit-elle. Amamellen attendit la nuit et cassa tout dans le campement. Le lendemain sa femme se désespéra de ne même plus avoir une écuelle pour boire du lait et demanda à Amamellen d'aller chercher les inaden» (Azawagh, 1999) ${ }^{32}$.

Edmond Bernus (1983: 243) rapporte également la même légende en précisant que la femme d'Amamellen dut boire le lait directement aux pis des animaux. Cet épisode nous amène à évoquer une deuxième légende qui concerne cette fois les ijobarren, géants rustiques qui vivaient aux temps préhistoriques, c'est-à-dire avant l'arrivée de la civilisation.

30. Dans l'ouest, les femmes nobles (timajeghen) ne réalisent pas (ou plus) le vélum de leur tente, car, à la différence de nombreux autres groupes, la tente est aujourd'hui la propriété de l'homme. C'est lui, lors du mariage, qui se la procure, le plus souvent auprès des femmes de groupes tributaires.

31. Informations relevées dans l'Arabanda pendant l'année 2000.

32. Une version tout à fait similaire à celle-ci a été relevée par Candalerio Saenz (1991: 68) auprès des inaden de la région de l'Ayr 
«Les ijobarren vivaient lorsque les pierres étaient encore molles. Ils n’avaient pas de lit, pas de coussins et buvaient à même le pis des animaux. Quand ils avaient faim, ils tuaient une girafe et la mangeaient toute crue» (Arabanda, 1999).

Ces deux légendes ont ceci en commun d'insister sur la valeur civilisatrice des techniques par les objets qu'elles permettent de créer. Dans un monde sans artisans ou sans techniques, les membres de la société sont en proie à l'inconfort, voire à la sauvagerie. Les techniques de fabrication permettent de passer d'un univers à un autre. Elles permettent d'établir une transition entre l'univers matériel d'avant et l'univers matériel actuel. Selon la pensée touarègue construite sur des couples d'oppositions symboliques (Claudot \& Hawad 1984), les techniques rendent possible le passage entre ces deux moments. L'évolution de l'homme n'est pas conçue comme une évolution rectiligne, sous-tendue par une suite de perfectionnements techniques, mais est au contraire construite autour de deux moments, qui s'opposent et se complètent de sorte que l'un n'a d'existence que par rapport à l'autre. Les techniques de fabrication ont un caractère dynamique, stimulant qui rend possible la civilisation.

Dans ce sens, bien des récits mettent en évidence une aptitude différente, une compétence particulière des inaden dans la réalisation d'artefacts :

«La couture d'une ténadt ne ressemble pas à celle de quelqu'un d'autre. La peau et la manière de colorer ne ressemblent pas à celle de quelqu'un d'autre" (Azawagh, 1999).

Si ce type de récits insiste, en premier lieu, sur le monopole qu'exercent les inaden dans le domaine technique, il suggère également qu'ils ont la faculté de mobiliser leurs connaissances pour fabriquer des objets, faculté que ne détiennent pas les autres catégories sociales.

Ces représentations s'appliquent également, me semble-t-il, au domaine connexe de la définition des conceptions esthétiques. La matière première (bois, métal, peau), qui appartient au monde de l'extérieur, possède à l'état brut des qualités esthétiques. La matière dicte, d'une certaine façon, des choix techniques et esthétiques opérés lors de la réalisation. Ainsi, la qualité du pelage d'un animal impose à la cordonnière de réaliser un sac tanné, mais non épilé, pour mettre en valeur la qualité de la toison. La couleur d'une essence d'arbre impose à un boisselier de ne pas noircir au feu une écuelle pour laisser apparaitre et apprécier la qualité du bois. Le travail de l'énad est, dans cette perspective, de mettre en valeur la beauté de la matière lors de la fabrication d'objets. Si les artefacts appartiennent au monde de l'intérieur, la matière qui les constitue est associée, elle, au monde de l'extérieur. L'énad, par ses connaissances techniques et esthétiques, rend possible ce passage de la matière d'un univers à un autre. En fabriquant des objets, il donne à la matière une sociabilité. Il y parvient en utilisant les potentialités techniques et esthétiques de celle-ci. 
Les représentations liées à la technique mettent en évidence la faculté de cette activité à établir des transitions entre les deux univers symboliques de l'intérieur et de l'extérieur. Les rites, les fonctions diplomatiques et sociales auxquelles participent les inaden sont également des activités qui permettent le passage entre deux moments, deux situations, deux étapes. Les inaden, en cumulant des activités techniques et sociales, ne détiennent en fait qu'un même type d'activités, celles qui permettent le passage entre deux mondes. Les nobles (imushagh) et les tributaires (imghad) appartiennent au cœur de la société et ne sont donc statutairement pas aptes à endosser les fonctions d'intermédiaire. Par contre les inaden, qui appartiennent aux marges de celle-ci, sont plus que toute autre catégorie sociale en mesure de jouer ce rôle. L'énad est donc un intermédiaire total, puisque dans toutes les activités qu'il exerce (dans le domaine social, politique, rituel mais aussi technique), il permet le passage, l'évolution, la transformation d'un état à un autre.

Cette analyse, fondée sur l'étude et l'interprétation de récits oraux confrontés à des données ethnographiques nouvelles, fait ressortir que la pratique d'une technique ne suffit pas à justifier le statut des inaden au sein de la société touarègue.

Les récits sur l'origine de cette catégorie sociale ont en commun, en utilisant des arguments divers, d'insister sur la proximité et en même temps sur la différence qui existent entre les nobles, les tributaires, d'une part, et les inaden, de l'autre. Ils n'évoquent qu'occasionnellement l'enjeu que représente la pratique d'une activité technique. Les mythes relatant la construction de la société et qui ont notamment comme fonction d'expliquer, voire de légitimer le statut actuel des inaden ne tiennent que rarement compte de l'activité technique qu'ils occupent.

Si certains inaden forment bien une communauté de métier, possédant des connaissances spécifiques, ils se distinguent, par exemple, des mállem, groupes de maitres artisans, essentiellement par la nature des activités qu'ils exercent au plan social. Leur statut d'intermédiaire au plan symbolique implique de n'exercer que les activités en correspondance avec celuici. Dans les représentations touarègues, les techniques permettent d'établir une transition entre les univers symboliques de l'intérieur et de l'extérieur. Elles ont en cela une valeur « civilisatrice " ou plutôt de socialisation de la matière brute. L'énad, dont la fonction symbolique est celui d'assurer un passage entre deux mondes, est, plus qu'une autre catégorie sociale, à même d'exercer les activités techniques. 
Loin de disqualifier pour la fonction sociale et politique, la pratique d'une technique ou l'acquisition d'un métier est au contraire en adéquation avec celle-ci. L'opposition statutaire entre les inaden et les nobles n'est pas fondée sur l'opposition entre le politique et les techniques. Si les activités de ces deux catégories sociales sont distinctes, elles ne s'opposent pas formellement comme s'opposent les fonctions sociales des nobles et des inaden.

L'énad, s'il peut aussi être un homme de métier, est d'abord et avant tout un intermédiaire social et symbolique ; la traduction de ce terme par «artisan» devient, dans cette perspective, extrêmement délicate.

MOTS CLÉSIKEYWORDS: Touareg/Twareg - Berbère/Berber - Sahara - artisan/craftsman technique/ technology - statut/status - métier/trade - mythe/ myth.

BIBLIOGRAPHIE

Agabi

1997 "Les forgerons du Maghreb", Encyclopédie berbère 19: 2889-2891.

Alojaly, Ghubeyd ag

1980 Lexique touareg-français. Copenhague, Akademisk Forlag.

Badi, Dida

2002 "Le voyage comme fondation de tribu dans la tradition orale des Ifughas ", in Hélène Claudot-Hawad, ed., Voyager d'un point de vue nomade. Paris, Paris-

Méditerranée/Aix-en-Provence, IREMAM : 53-61.

\section{Benfoughal, Tatiana}

1996 « Du palmier dattier à la matière plastique. Tradition et mode dans la fabrication des vanneries sahariennes ", in Hélène Claudot-Hawad, ed., Touaregs et autres Sahariens entre plusieurs mondes. Aix-enProvence, Edisud : 57-78.

\section{Benhazera, Maurice}

1908 Six mois chez les Touaregs du Ahaggar. Alger, A. Jourdan.

Bernus, Edmond

1983 «Place et rôle du forgeron dans la société touarègue", in Nicole Échard, ed., Métallurgies africaines. Nouvelles contribu- tions. Paris, Société des africanistes

(«Mémoires » 9) : 237-251.

\section{Bernus, Suzanne}

1983 «Hypothèses sur le processus de constitution d'une tawshet, l'exemple des imeghad Illabaken ", in Suzanne Bernus et al., eds, Le Fils et le neveu: jeux et enjeux de la parenté touarègue. Cambridge, Cambridge University Press/Paris, Éd. de la Maison des sciences de l'homme: 129-157.

\section{Boëtsch, Gilles \& Jean-Noël Ferrié}

2000 «De la modernité paradoxale du point de vue de l'anthropologie physique sur les groupes serviles au Sahara ", in Mariella Villasante-de Beauvais, ed., Groupes serviles au Sahara. Paris, CNRS Éditions : 269-276.

Bonte, Pierre

1985 «Faits techniques et valeurs sociales: quelques directions de recherche", Techniques et cultures $5: 19-48$.

1998 L'Émirat de l'Adrar. Histoire et anthropologie d'une société tribale du Sahara occidental. Paris, EHESS, thèse de doctorat d'État.

\section{Bourgeot, André}

1993 «De l'identité à l'ethnicité : le cas des Touaregs ", La Pensée 296 : 49-58. 


\section{Bromberger, Christian}

1988 "Comment peut-on être Rašti? Contenus, perceptions et implications du fait ethnique dans le Nord de l'Iran ", in Jean-Pierre Digard, ed., Le Fait ethnique en Iran et en Afghanistan. Paris, Éditions du CNRS : 89-107.

\section{Camps, Gabriel}

1982 «Réflexions sur l'origine des juifs des régions nord-sahariennes ", in Michel Abitbol, ed., Communautés juives des marges sahariennes du Maghreb. Jérusalem, Institut Ben-Zvi : 57-67.

1991 "Bavares", Encyclopédie berbère 9 : 1394-1399.

1995 Les Berbères. Mémoire et identité.

Paris, Errance.

\section{Casajus, Dominique}

1987a La Tente dans la solitude. La société et les morts chez les Touaregs Kel Ferwan. Paris, Éditions de la MSH/Cambridge, Cambridge University Press.

1987b «Crafts and Ceremonies: The Inaden in Tuareg Society ", in The Other Nomades. Peripatetic Minorities in CrossCultural Perspectives. Cologne-Vienne, Aparma : 291-310.

\section{Claudot-Hawad, Hélène}

1985 "Adghagh. Histoire du peuplement", Encyclopédie berbère 2 : 147-153.

1987 "Amamellen", Encyclopédie berbère $4: 556-558$.

1993 Les Touaregs. Portrait en fragments. Aix-en-Provence, Edisud.

1996a "Identité et altérité d'un point de vue touareg ", in Hélène Claudot-Hawad, ed., Touaregs et autres Sahariens entre plusieurs mondes. Aix-en-Provence, Edisud : 7-16.

1996b «Personnages de l'entre-deux chez les Touaregs. L'initié, l'énad, l'aggag, le soufi... ", in Hélène Claudot-Hawad, ed., Touaregs et autres Sahariens entre plusieurs mondes. Aix-en-Provence, Edisud : 223-238.

1998 "La hiérarchie des savoirs et des pouvoirs dans la société touarègue précoloniale et la recomposition des rôles socio-politiques pendant la guerre anticoloniale et après la défaite", Nomadic Peoples 2 (1-2) : 17-38.

2000 "Captif sauvage, esclave enfant, affranchi cousin. La mobilité statutaire chez les Touaregs (imajaghen)", in Mariella Villasante-de Beauvais, ed., Groupes serviles au Sahara. Paris, CNRS Éditions : 237-268.

\section{Claudot, Hélène \& Mahmoudan Hawad}

1984 "Ebawellessuf. Les notions d'intérieur et d'extérieur dans la société touarègue", Revue de l'Occident musulman et de la Méditerranée 38 : 171-179.

1987 "Amahagh, amajagh, amashagh", Encyclopédie berbère 4 : 554-555.

\section{Detienne, Marcel}

1981 L'Invention de la mythologie. Paris, Gallimard.

\section{Dumont, Louis}

1966 Homo hierarchicus. Le système de castes et ses implications. Paris, Gallimard.

\section{El Alaoui, Narjys}

2001 Le Soleil, la lune et la fiancée végétale. Essai d'anthropologie des rituels. Aix-enProvence, Edisud.

\section{El Bekri}

1913 Description de l'Afrique septentrionale. Alger, A. Jourdan.

Foucauld, Charles de

1951-1952 Dictionnaire touareg-français. Paris, Imprimerie nationale.

Hincker, Catherine

1996 «Forgerons touaregs et métallurgistes de l'Udalen. Un exemple de complémentarité technique ", in Hélène Claudot-Hawad, ed., Touaregs et autres Sahariens entre plusieurs mondes. Aix-en-Provence, Edisud: 50-56.

2002 « Matière et métier. Le travail des peaux et du cuir chez les Touaregs de l'Ouest (Mali) ", in Sylvie Beyries \& Frédérique Audoin-Rouzeau, eds, Le Travail du cuir de la préhistoire à nos jours. Antibes, Association 
pour la promotion et la diffusion des connaissance archéologiques : $99-112$.

\section{Jemma, Danièle}

1971 Les Tanneurs de Marrakech. Alger, CNRS ("Mémoire du Centre de recherches anthropologiques, préhistoriques et ethnographiques" 19).

1972 "Les artisans de l'Ahaggar ", Libyca 20 : 269-290.

\section{Jonckers, Danielle}

1979 « Notes sur le forgeron, la forge et les métaux en pays minyaka ", Journal des Africanistes 49 (1) : 103-124.

\section{Lemonnier, Pierre}

1991 "Technique (système) ", in Pierre Bonte \& Michel Izard, eds, Dictionnaire de l'ethnologie et de l'anthropologie. Paris, PUF : 697-698.

\section{Lewicki, Tadeusz}

1978 "L'origine nord-africaine des Bafour ", Actes du II congrès international d'études des cultures de la Méditerranée occidentale, Malte, 1976. Barcelone, Universidad autonoma : 145-153.

\section{Lhote, Henri}

1984 Les Touaregs du Hoggar. Paris, Armand Colin.

\section{Monteil, Charles}

1951 «Problèmes du Soudan occidental : juifs et judaïsés ", Héspéris 38 (3-4) : 265-298.

Nicolaisen, Johannes

1962 "Afrikanske smede", Kulm: 33-79.

Nicolas, Francis

1951 «Textes ethnographiques de la "Tamâjeq" des Iullemmeden de l'est.
Touâreg de la colonie du Niger, Afrique occidentale de l'Est ", Anthropos 46 :

755-800.

\section{Norris, Harry T.}

1972 Saharan Myth and Saga. Oxford, Clarendon Press.

1975 The Tuaregs. Their Islamic Legacy and its Diffusion in the Sahel. Warminster, Aris \& Phillips.

Oliel, Jacob

1994 Les Juifs du Sahara. Le Touat au moyen-âge. Paris, CNRS Éditions.

\section{Rasmussen, Suzann}

1992 "Ritual Specialists, Ambiguity and Power in Twareg Society ", Man 27 (1) : 105-128.

\section{Saenz, Candalerio}

1980 «Kinship and Social Organisation of the Inaden ", communication à la Table ronde sur la parenté touarègue, Gif-surYvette, CNRS, 23-26 septembre, multigr. 1991 They Have Eaten our Grand Father. The Special Status of the Twareg Smiths. New York, Columbia University, Ph.D. thesis.

Schroeter, Daniel J.

1997 «La découverte des juifs berbères », in Michel Abitbol, ed., Relations judéo-musulmanes au Maroc: perceptions et réalité. Paris, Stavit : 169-187.

Tamari, Tal

1997 Les Castes de l'Afrique occidentale. Artisans et musiciens endogames. Nanterre, Société d'ethnologie.

\section{Vernant, Jean-Pierre}

1952 «Prométhée et la fonction technique ", Journal de psychologie normale et pathologique 45 : 419-429. 
Catherine Hincker, Identité et métier des Inaḍen. La valeur sociale des techniques chez les Touaregs de l'Ouest. - En s'appuyant sur des sources orales nouvelles recueillies lors d'enquêtes de terrain à l'ouest du Sahara, l'auteur revient sur la question du statut dans la société touarègue de ceux qu'on appelle couramment en français "artisans». Les membres de cette catégorie sociale (inaden) exercent le plus souvent deux activités distinctes : un rôle d'intermédiaire sur le plan social, politique et rituel ainsi qu'une activité technique. Quelles représentations les Touaregs associent-ils à ces deux activités et sont-elles à l'origine du statut des " artisans » ? L'analyse des mythes montre que l'origine juive des artisans (inaden) est un stéréotype construit pour signaler la différence entre les membres de cette catégorie et le reste de la société (nobles ou tributaires). Dans les conceptions touarègues, la technique tout comme les rites permettent de passer d'un état, d'un univers symbolique à un autre. Seuls ceux qui ont le statut d'intermédiaire social et symbolique peuvent exercer ces activités.
Catherine Hincker, Inaden's Identity and Craft: The Social Value of Techniques among the Western Tuareg. - New data from oral sources gathered during fieldwork in the western Sahara are used to reexamine the question of the status of craftsmen (inaden) in Tuareg society. Persons in this social category usually have two distinct activities, namely as middlemen on the social, political and ritual levels and as persons exercising a technical activity. What ideas do the Tuareg associate with these two activities? Do these ideas lie at the origin of the status of craftsmen? The analysis of myths shows that the Jewish origin of craftsmen is a stereotype constructed for pointing out the difference between inaden and the rest of society (nobles or wards). According to Tuareg conceptions, techniques, like rites, provide for a passage from one state, or one symbolic universe, to another. Only those persons who have the status of social and symbolic middlemen can exercise these activities. 